\title{
Condiciones ambientales relacionadas con los deterioros de obras ubicadas en patio del Convento de San Francisco de Quito
}

\section{Environmental conditions related to the deterioration of the easel paintings located in the main cloister of the Convent of San Francisco de Quito}

\author{
Valeria Catalina Villamar Manzano, investigadora independiente (valeria_vm@hotmail.com) \\ Julio Enrique Benítez Telles, Universidad Tecnológica Equinoccial (jbenitez@ute.edu.ec) \\ Recibido: 2016 - $10-28 \quad$ Aceptado: 2017 - $04-17$
}

\begin{abstract}
Resumen
Las alteraciones perceptibles en pintura al óleo resultan de la diferente reacción que presentan los materiales constitutivos de la obra al entrar en contacto con el entorno. El presente trabajo forma parte de una serie de investigaciones que buscan aportar evidencia empírica, a favor o en contra, de diferentes teorías que se han utilizado para explicar fenómenos relacionados con la alteración del patrimonio cultural. Usualmente, las obras pictóricas se exponen en espacios cerrados donde se pueden mantener las condiciones ambientales dentro de ciertos rangos, para reducir así las tensiones internas y hacer más lentos los procesos de alteración. Sin embargo, en el caso que nos ocupa, las obras se encuentran en espacios abiertos donde es prácticamente imposible controlar los factores climáticos. La finalidad de esta investigación fue verificar el grado de relación entre las condiciones ambientales de humedad relativa (HR), temperatura (t) e iluminancia (E) existentes en el patio del claustro principal del Convento de San Francisco de Quito, con los indicadores de deterioro presentes en 13 pinturas de caballete expuestas en este mismo espacio. Los valores que alcanzan los tres factores fueron capturados con sensores remotos colocados junto a esas obras, mientras que las alteraciones que presentan las obras fueron registradas utilizando una ficha específica. Como resultado se obtuvo que existe una relación directa donde el deterioro más común fue el craquelado. La humedad relativa es el factor de mayor incidencia; además, todos los factores se encuentran fuera de los límites ideales para la conservación de obras pictóricas sobre tela.
\end{abstract}

Palabras claves: pintura de caballete, agentes e indicadores de deterioro, humedad relativa, temperatura, iluminancia.

\begin{abstract}
The perceptible alterations in oil painting (cracking, fissures, rising, colorfading, etc.) are the result of the differentreactions of the constitutive materialsof the artpiecewhen they comeinto contactwith theenvironment. The present work is part of a series of investigations that seek to provide empirical evidence, for or against, different theories that have been used to explain phenomena related to the alteration of cultural heritage; the ultimate goal, is to improve conservation processes to reduce the number of direct interventions and to slow down the processes of deterioration of matter. Usually, paintings are exbibited in enclosed spaces such as museums or galleries, where environmental conditions can be maintained within certain ranges, thus reducing internal tensions and slowing the processes of alteration. However, in the case at hand, the artworks are in open spaces where it is practically impossible to control the climatic factors. The purpose of this research was to verify the degree of relationship between the environmental conditions of relative humidity $(R H)$, temperature ( $t$ ) andluminance (E) in the courtyard of the maincloister of the Convent of San Francisco de Quito, with deterioration indicatorspresent in 13easel paintings exposed in thissamespace. The values that reach the three factors indicated above (HR, $t$ and E) were captured with remote
\end{abstract}


sensors placed next to the paintings exposed in the corridors of the main cloister. Mean while, the alterations presented on them were registered using a specific form modified for this purpose. As a result it was obtained that there is a direct relationship, where the most common deterioration was cracking, followed by peeling and fissures. The relative bumidity is the factor of greater incidence, followed by the temperature and finally the luminance. Furthermore, in this space, all factors are outside the ideal limits for the conservation of pictorial works on canvas.

Keywords: easel painting, agents and indicators of deterioration, relative bumidity, temperature, light intensity.

\section{INTRODUCCIÓN}

Desde los años setenta, autores como Marion Mecklenburg, Gerry Hedley, entre otros (Keyser, 1984) iniciaron investigaciones sobre los procesos de alteración en pintura al óleo, trabajando sobre probetas sometidas a la acción de tres factores: humedad relativa, temperatura e iluminancia, cuyos resultados después eran comparados con las alteraciones, atribuidas a los mismos tres factores, que se observaban en pinturas antiguas.

Las probetas empleadas en dichos estudios replicaron los tres estratos constitutivos de las obras pictóricas: 1. Soporte, 2. Base de preparación y3. Capa pictórica. Los ensayos al aire libre fueron efectuados en las latitudes templadas y aquellos en laboratorio considerando las condiciones climáticas de las mismas zonas geográficas. Y, finalmente, los resultados se compararon con alteraciones reportadas en pinturas expuestas oalmacenadas en Europa o Estados Unidos.

A diferencia de la variables consideradas por Mecklenburg, Hedley, Tumosa o Roche, en la ciudad de Quito, la humedad relativa y la temperatura pueden variar en un solo día entre $20-25 \%$ y $15-18^{\circ} \mathrm{C}$ respectivamentey las bases de preparación presentes en las obras motivo de este estudio están compuestas de aceite con sulfato de calcio yóxido de hierro. Porlo tanto, el comportamiento de los materiales deberíaser también diferente alos reportados por dichos autores.

El trabajo que se expone a continuación se planteó con el fin de establecer en qué medida se encuentran los factores ambientales (humedad relativa, temperatura e iluminancia) fuera de los parámetros ideales para la conservación que rodean las obras expuestas en los corredores del claustro principal del convento San Francisco y relacionarlos con las alteraciones presentes en los estratos pictóricos de las obras expuestas en este mismo espacio.

\section{Materiales pictóricos y factores de alteración}

Usualmente, la pintura de caballete está constituida por tres capas poliméricas principales: soporte (celulosa), base de preparación (aglutinante con carga mineral) y capa pictórica (aglutinante con pigmento). Cada uno de estos estratos está a su vez compuesto por materiales de estructura cristalina o amorfa, de origen natural o sintético, de naturaleza orgánica o inorgánica, con mayor o menor afinidad con el agua, y, que para ser empleados, pasaron por etapas de extracción, refinamiento y manufactura según los fines que deberían cumplir. Una vez obtenido el material, este busca retornar a un estado anterior y de mayor estabilidad: por un lado, se inician procesos internos de envejecimiento, y luego, entra en interacción con factores presentes en el entorno de la obra; resultando en alteraciones siempre inevitables e irreversibles (por principio de termodinámica) que pueden ir desde ligeras variaciones de la apariencia, como el desvanecimiento del color, hasta la falla total de las propiedades mecánicas por rotura de fibras (Czichos, Saito y Smith, 2006)

De los diferentes factores de alteración que afectan a la pintura al óleo, la literatura tradicionalmente se ha centrado en tres: humedad relativa (HR), temperatura (t) e iluminancia (E), por ser los factores que siempre están presentes y no se los puede eliminar de los espacios de exposición y almacenaje. Cantidades inadecuadas de estos factores y sus bruscas fluctuaciones provocan la mayoría de alteraciones irreversibles de la pintura al óleo; si estos factores se encuentran dentro de determinados límites $\left(50-60 \% \mathrm{HR}, 16-20^{\circ} \mathrm{C}\right.$ y máximo 180lx), como ocurre en museos o salas de exposición, el resultado de la interacción es menory los cambios por envejecimiento se ralentizan (Roche, 2005; Martiarena, 1992; Herráez y Rodríguez, 1989).

Lo contrario ocurre en pinturas expuestas en ambientes abiertos, donde es difícil controlar la cantidad e intensidad de estos tres factores, en cuyo caso, las alteraciones se espera sean significativamente más marcadas. Condiciones adversas de este tipo ocurren en la ciudad de Quito donde, entre 2006 y 2012, se registraron medias mínimas y máxima de 27,9\%HR - 99,2\%HR y 
$6,5^{\circ} \mathrm{C}-28,3^{\circ} \mathrm{C}$ (Instituto Nacional de Meteorología e Hidrología - INAMHI-, 2006, 2007, 2008, 2009, 2010, 2011, 2012) y pueden ser responsables de algunas alteraciones como desvanecimiento del color (Saltos, 2014) o la formación de una capa iridiscente en la superficie pictórica (Vega, 2004).

\section{Procesos de alteración}

Si bien es difícil extrapolar los resultados obtenidos en laboratorio debido a: las diferencias entre los factores de alteración involucrados, las características geográficas donde se desarrolla el estudio, o el grosor y color de las capas polícromas, entre otros, algunos principios físicos y químicos nos dan pistas para establecer explicaciones tentativas a los fenómenos ligados a los procesos de alteración de pintura al óleo expuestas en espacios abiertos, así:

Sabemos que la cantidad de vapor de agua que estará en contacto con una obra pictórica es inversamente proporcional a la temperatura y directamente proporcional a la presión atmosférica. En consecuencia, el incremento de la temperatura durante el día provocará, en primer lugar, , sequedad en el aire y la merma de los materiales hidrófilos; y luego, la dilatación de los materiales minerales, principalmente los metálicos. Lo contrario ocurrirá en la noche, cuando baja la temperatura: los compuestos minerales se contraerán, habrá un aumento en la humedad relativa y los materiales hidrófilos se hincharán.

Estos movimientos generan en el interior de los estratos pictóricos fuerzas que, dentro de los límites elásticos de cada material, deforman de manera reversible cada estrato - fase elástica-; cuando las fuerzas exceden el límite elástico del material, la deformación es irreversible - fase plástica-, puede sobrevenir la rotura del estrato al incrementarse el valor de las fuerzas o por la fatiga del material.

Por lo general, la apariencia y resistencia de los materiales que constituyen los estratos pictóricos se ven afectadas por procesos de fotoxidación porque, al ser bastante baja la energía de unión entre moléculas que conforman los estratos, la radiación solar, en especial el espectro del ultra violeta (UV) es suficiente para debilitar los enlaces y romperlos. Al momento de quelas moléculas absorben fotones quedan excitadas electrónicamente y desequilibradas térmicamente, y buscarán liberarse de la energía recibida tan pronto puedan, hecho que ocurre cuando la radiación deja de incidir sobre la pintura y esta libera al entorno la energía en forma de calor. Cuando la energía recibida no es suficiente para romper la unión de la molécula, esta la libera, en unos casos como calor, en otros como fluorescencia, y en otros como fosforescencia; no obstante, la unión queda en un estado de transición fotosensible y finalmente se romperá por efecto acumulativo en la unión más débil, resultado en dos radicales que se recombinarán o interactuarán con otras moléculas presentes en su entorno, e.g. $\mathrm{O}_{2}, \mathrm{H}_{2} \mathrm{O}, \mathrm{CO}_{2}$, etc. para formar productos finales mâs estables (Czichos et al., 2006).

Roche ( 2005), Mecklenburg (2007) y Michalski (2009) coinciden en señalar que la variación volumétrica provocada por las fluctuaciones de humedad relativa es mayor que aquella producida por incremento o reducción de temperatura, y que, cuanto más hidrófilos sean los materiales, mayores serán su hinchazón y merma; mientras que los materiales hidrófobos no presentan cambios volumétricos de consideración (Mecklenburg, 2007). La diferencia en el comportamiento de los materiales generan fuerzas opuestas en las zonas de unión de los estratos, los cuales, por sus características elásticas pueden absorber parcialmente dichas fuerzas las características elásticas de las capas poliméricas, las variaciones constantes (en especial aquellas drásticas), provocan la fatiga de los materiales y el desplazamiento de la fase elástica a la plástica, en la cual, las capas dejan de ser flexibles y se tornan rígidas y quebradizas.

En 2007, Mecklenburg observó que entre 10\%HR y $60 \% \mathrm{HR}$ las fibras de lino se ven sometidas a fuerzas de escasa intensidad; a partir de $70 \% \mathrm{HR}$ dichas fuerzas tienen un incremento gradual, $y$ al sobrepasar el 75\%HR (Michalski, 2009), 80\%HR (Mecklenburg, 2007), se produce un encogimiento muy importante de la tela porque al aumentar el volumen transversal de las fibras, aquellas de urdimbre deben recorrer mayor espacio y tensado la telay las fuerzas se incrementan deforma significativa.

La capa pictórica, más bien hidrófoba pero flexible por estar compuesta por aceite, puede absorber parcialmente la merma, hinchazón y tensión de la tela sin embargo, serán los pigmentos empleados quienes determinen cuan flexible es este estrato: Mecklenburg (2007) confirmó que con pigmentos tierra la capa pictóricar es más flexible que aquella formada por blanco de plomo o malaquita y el mismo aglutinante, y que, la baja humedad relativa incrementa la rigidez de la pintura, tornándose extremadamente quebradiza; y en 2009, Michalski observó que al sobrepasar el $75 \% \mathrm{HR}$, las pinturas al óleo comienzan a craquelarse.

Czichos et al. (2006) en un resumen sobre el efecto de la radiación solar sobre capas poliméricas, indica 
que en 1980, Boysen constató que pinturas oleosas expuestas a radiación UV pierden brillo conforme aumenta la temperatura del ambiente; un año después, Furneagux, Ledbury y Davis concluyeron que los procesos de foto oxidación se ven afectados por la cantidad de oxígeno puro existente en el ambiente; para 1982, Kämpfy Papenroth confirmaron que los pigmentos sensibles se desvanecen de forma muy marcada cuando son expuestos a la radiación visible del sol; en 1988, Trubiroha documentó que los ambientes húmedos están relacionados con el amarillamiento de las capas translúcidas y ralentiza sus alteraciones; y en 2004, Trubiroha, Geburting y Wachtendorf demostraron que su efecto se ve condicionado por el tiempo de exposición, la temperatura, el grado de cristalinidad y espesor de la capa pictórica (en Czichos et al. 2006 ).

\section{METODOLOGÍA}

\section{Objeto de estudio}

Trece pinturas de caballete de gran formato $(238,4 \mathrm{x}$ $246,8 \mathrm{~cm}$ en promedio), todas ejecutadas con la técnica deóleo sobrelienzo que datan delos siglos XVIIyXVIII. Todas estas obras se encuentran expuestas en el patio del claustro principal del Convento, en la planta baja referentes ala vida de San Francisco deAsís distribuidas en las paredes Nordeste (NE), Sudeste (SE), Sudoeste (SO) y Noroeste (NO); y 6 en la planta alta relativas a la vida de Jesús, todas distribuidas en la pared NE.

\section{Instrumentos}

Los factores ambientales se midieron con sensores remotos de la marca Onset HOBO (U12-012), programándolos para medir humedad relativa, temperatura e iluminancia cada 15 minutos. Los valores registrados se analizaron con el programa de procesamiento de datos Microsoft Excel 2010.

Los deterioros presentes en las obras se registraron en una ficha específica, basada en la desarrollada y propuesta por Saltos (2014).

La valoración de las alteraciones se realizó en función de: la complejidad de un posible tratamiento en caso de ser intervenido, y de la cantidad de superficie afectada, este valor se ajustó para obtener tres niveles de alteración que se corresponden con las valoraciones de bueno, regular y malo.

Se consideraron como deterioros de nivel 1 aquellos cuya presencia no implica una pérdida total del bien, y que el tratamiento para eliminarlos no es fundamental o imperativo; e.g. polvo, suciedad. El nivel 2 agrupó las alteraciones que requieren de intervención, pero que, si se controla el entorno las operaciones pueden esperar; e.g. deformaciones, ondulaciones.

Finalmente, como deterioro de nivel 3 se consideraron aquellos que ponen en riesgo la permanencia material del objeto en el estado presente, su intervención es imperativa y probablemente no reversible; p.e. roturas, faltantes.

\section{Procedimientos}

Las obras estudiadas fueron codificadas con un sistema alfanumérico específico para identificarlas según los requerimientos de este estudio.

El período de estudio corrió entre septiembre de 2014 y marzo de 2015, y fue establecido considerando los valores mínimos y máximos anuales de cada factor consignados en los anuarios del INAMHI entre 2006 y 2012.

Se instalaron 6 sensores remotos, de las características indicadas en el apartado anterior, en las mismas paredes donde se encontraban las obras, 4 en la planta baja, uno porcada pared NE, SE,SOyNO,y2 en la pared NE de la planta alta. Todos los sensores se instalaron a una altura de $2,66 \mathrm{~m}$, cerca de las obras estudiadas.

Los valores registrados por los sensores (más de 100.000) se migraron a un programa de procesamiento de datos Microsoft Excel 2010 para su análisis, se contabilizaron cuántas veces, y en qué medida, los valores de HR, $t$ y E se encontraban fuera de los parámetros considerados como ideales para conservar pintura sobre tela.

La ficha de registro y estado de conservación para pintura de caballete consta de 2 partes: en la primera se apuntaron datos generales de identificación de la obra; y en la segunda, se describieron los deterioros observados en cada una de ellas. Esta ficha se diseñó a partir de una anterior que fue probada por Saltos en 2014.

La determinación del estado de conservación se calculó en función del área afectada por cada uno de los deterioros observados y de la complejidad que implicaría una posible intervención; este resultado fue ajustado con un factor $n$ para luego clasificar el estado de las obras en bueno, regular o malo. 


\section{RESULTADOS}

\section{Condiciones ambientales y frecuencia}

Las medias de los valores absolutos de cada factor: general, mínimo y máximo, y el "peso" de cada uno en función de la frecuencia en que los estratos estuvieron por debajo, sobre o dentro de las condiciones adecuadas, se resumen en la Tabla 1. El valor promedio para las mínimas absolutas de iluminancia no se calcularon porque se considera adecuado para la conservación entre 0 y 180lx.

Tabla 1. Promedio de valores absolutos por agente de alteración y frecuencia de los mismos (f\%)

\begin{tabular}{|c|c|c|c|c|}
\hline Factor & Promedio General & $\begin{array}{c}\text { Valores ideales } \\
\text { (f. en este rango) }\end{array}$ & $\begin{array}{c}\text { Media de máximos } \\
\text { (f. en este rango) }\end{array}$ & $\begin{array}{c}\text { Media de mínimos } \\
\text { (f. en este rango) }\end{array}$ \\
\hline Humedad relativa & $65,24 \% \mathrm{HR}$ & $65,24 \% \mathrm{HR}(19,42 \%)$ & $86,54 \% \mathrm{HR} \quad(65,71 \%)$ & $28,22 \% \mathrm{HR}(14,80 \%)$ \\
\hline Temperatura & $15,65^{\circ} \mathrm{C}$ & $16-20 \mathrm{C}(33,86 \%)$ & $23,27 \mathrm{C} \quad(3,89 \%)$ & $10,80^{\circ} \mathrm{C}(62,25 \%)$ \\
\hline Iluminancia & $486,99 \mathrm{l} \mathrm{x}$ & $486,991 \mathrm{x}(61,23 \%)$ & $5167,781 \mathrm{x}(38,77 \%)$ & $-(68,23 \%)$ \\
\hline
\end{tabular}

Autor: elaboración propia

Los valores promedio de la humedad relativa e iluminancia están fuera de los parámetros considerados ideales para que los procesos de alteración sean mínimos, mientras que la temperatura promedio registrada puede asumirse comoadecuada.

Humedad relativa, el 14,80\% de veces este factor permaneció bajo el mínimo ideal $(50 \% \mathrm{HR})$, el $19,42 \%$ de veces entre los 50 y $60 \% \mathrm{HR}$, el 54,04\% de veces la HR estuvo entre $80 \%$ HR y el máximo ideal $(60 \% \mathrm{HR})$, y un $11,74 \%$ de veces los valores máximos fueron superiores a $80 \% \mathrm{HR}$ (límite sobre el cual las fibras hinchan transversalmente).
Temperatura, el $62,25 \%$ de veces estas estuvieron por debajo delaideal $\left(16^{\circ} \mathrm{C}\right)$, franja dondela capa pictórica tiende a perder elasticidad. Un 3,89\% de veces que superaron los $20^{\circ} \mathrm{C}$ (el máximo recomendado). El restante $33,86 \%$ se mantuvo entre los 16 y los $20^{\circ} \mathrm{C}$

Iluminancia, el 38,77\% de veces, la cantidad de flujo luminoso que recibían las obras sobrepasó los 180lx recomendados para conservar pinturas al óleo. No obstante, debido a que el efecto de este factor es acumulativo y su elevada concentración, los procesos fotoquímicos son importantes, tal como se verá más adelante.

\section{Deterioros}

Tabla 2. Deterioros observados según niveles de conservación

\begin{tabular}{|c|c|c|c|c|c|c|c|c|c|c|c|}
\hline \multirow{2}{*}{$\begin{array}{c}\text { Código de la } \\
\text { pintura }\end{array}$} & \multicolumn{4}{|c|}{ NIVEL 1} & \multicolumn{3}{|c|}{ NIVEL 2} & \multicolumn{4}{|c|}{ NIVEL 3} \\
\hline & $A$ & $B$ & $C$ & D & $E$ & $\mathrm{~F}$ & $G$ & $\mathrm{H}$ & 1 & $\mathrm{~J}$ & $\mathrm{~K}$ \\
\hline PbSE1 & $x$ & $x$ & & & $x$ & & & $x$ & & & \\
\hline PbSE2 & $x$ & $x$ & $x$ & $x$ & $x$ & & & $x$ & $x$ & & $X$ \\
\hline PbSO1 & $x$ & $x$ & $x$ & $x$ & $x$ & & $x$ & & & $x$ & \\
\hline PbNO1 & $x$ & $x$ & $x$ & $x$ & $x$ & & & $x$ & $x$ & & $x$ \\
\hline PbNE1 & $x$ & $x$ & $x$ & $x$ & $x$ & & & $x$ & $x$ & & $x$ \\
\hline PbNE2 & $x$ & $x$ & $x$ & $x$ & $x$ & & $x$ & $x$ & $x$ & & $x$ \\
\hline PbNE3 & $x$ & $x$ & & $x$ & $x$ & $x$ & & $x$ & $x$ & & $x$ \\
\hline PaNE1 & $x$ & $x$ & $x$ & $x$ & & & & $x$ & $x$ & & $x$ \\
\hline PaNE2 & $x$ & $x$ & & $x$ & & $x$ & & $x$ & & $x$ & $x$ \\
\hline PaNE3 & $x$ & $x$ & $x$ & $x$ & & $x$ & & $x$ & $x$ & & $x$ \\
\hline PaNE4 & $x$ & $x$ & $x$ & $x$ & & $x$ & & $x$ & $x$ & & $x$ \\
\hline PaNE5 & $x$ & $x$ & $x$ & $x$ & & $x$ & & $x$ & $x$ & & \\
\hline PaNE6 & $x$ & $x$ & $x$ & $x$ & & $x$ & & & & & \\
\hline $\begin{array}{l}\text { A : Polvo } \\
\text { B : Suciedad } \\
\text { C: Grietas }\end{array}$ & & & $\begin{array}{l}D: \\
E: \\
F:\end{array}$ & $\begin{array}{l}\text { elad } \\
\text { neci } \\
\text { acio }\end{array}$ & & & $\begin{array}{l}\text { Levan } \\
\text { Despr } \\
\text { Rotu }\end{array}$ & $\begin{array}{l}\text { nto } \\
\text { niento }\end{array}$ & & $\begin{array}{l}J: F a \\
K: P\end{array}$ & lencia \\
\hline
\end{tabular}

Autor: elaboración propia 


\section{Nivel 1 de deterioro}

Todas las obras presentaron los mismos indicadores de Nivel 1: polvo y suciedad tanto por el anverso como por el reverso; chorreados de pintura; manchas de origen no identificado; $y$, solo por el reverso, nidos y telas de araña, arañas e insectos. En tres obras, grietas y craquelados no eran evidentes o se confundían con otras alteraciones. Este tipo de deterioro se evidencia mayormente en colores con mezcla de blanco de plomo como tonos blancos y apastelados de las obras analizadas.

\section{Nivel 2 de deterioro}

Desvanecimiento de color se observó en toda la superficie de las pinturas de la planta baja y no en las de la planta alta. Las ondulaciones están presentes en cinco de las seis obras de la planta alta y en una de la planta baja. Solo dos obras de la planta baja presentan levantamientos.

\section{Nivel 3 de deterioro}

Once obras presentan desprendimientos de capa pictórica indistintamente dela ubicación de la pintura. Roturas por el desgarre de fibras se evidenciaron en 9 cuadros. Dos obras presentaron faltantes de soporte, producto también del desgarre de fibras. Finalmente, 9 obras presentaban pulverulencia.

Bajo las condiciones de humedad relativa y temperatura antes indicadas, y aplicando las tablas sobre contenido de humedad $(\mathrm{CH})$ a diferentes temperatura y humedad relativa (Kollman y Côté, 1968), podemos estimar que el contenido promedio de humedad de la fibra sería de $12 \% \mathrm{CH}$, con un valor máximo de 18\%CH (para máxima humedad relativa y mínima temperatura) y un valor mínimo de $6 \% \mathrm{CH}$ (mínima humedad relativa y máxima temperatura). La oscilación entre estos valores conlleva procesos de hinchazón y merma volumétrica del 6\%. La cola empleada para el sisado tendrá un comportamiento similar, hinchando o mermando según aumente o disminuya la humedad relativa, pero la variación volumétrica sería de 3\%, según los valores medidos por Mecklenburg (2007).

Finalmente, la capa pictórica tendrá variaciones de volumen entre $0,5 \%$ para blanco de plomo y 3,0\% para tierras y ocres (Mecklenburg, 2007) Al hinchar la fibra, los hilos de trama y urdimbre se ven sometidos a tracción y distancia entre crestas y valles se reduce provocando compresión de las capas de preparación y pictórica; cuando sobrepasa el $80 \% H R$, las fibras pasan a fase plástica y pueden llegar a romperse. Al bajar la humedad, merma el volumen de la fibra y la tela se libera de fuerzas de tracción, pero las fibras no regresan a su estado anterior formando ondulaciones y las capas de preparación y pictórica poco elásticas se craquelarán.

Estos fenómenos concuerdan con los deterioros observados: la presencia de grietas se explicaría por la combinación de alta humedad relativa y baja temperatura, usuales en la noche y madrugada. Se produciría tensión en el soporte mientras las capas de pintura y base de preparación se tornarían rígidas, los constantes ciclos terminarían causando estas hendiduras en las capas más frágiles. Los Craquelados serían resultado del secado de los aceites o por el diferente comportamiento al incrementarse la HR (91,17\%HR máxima absoluta) yal reducirse la temperatura $\left(10,80^{\circ} \mathrm{C}\right.$ mínima absoluta).

En un nivel más avanzado del proceso ocurrirían los desprendimientos como resultado del diferente comportamiento del soporte y las capas superiores, temperatura y humedad relativa cambiantes, y el envejecimiento natural del aglutinante que ha perdido sus cualidades deadhesión y cohesión. Al momento de envejecer, el aceite perdió sus propiedades elásticas y no se adapta a los movimientos que experimenta el soporte con los incrementos de humedad relativa. La diferente intensidad de fuerzas provoca el levantamiento de los bordes de un craquelado. Los ciclos continuos terminan por desprender la capa de pintura limitada por las craqueladuras. Otro deterioro relacionado es la pulverulencia y se explica por la pérdida de elasticidad de los aglutinantes y por la reducción de su resistencia mecánica volviéndose la capa extremadamente quebradiza y deleznables.

\section{CONCLUSIÓN}

\section{Agentes ambientales de alteración}

Los promedios de cada factor no se ajustan a las condiciones óptimas: la humedad relativa supera a la ideal en más de 5\% (65,24\%), la temperatura se encuentrabajo la recomendable en $2,35^{\circ} \mathrm{C}\left(15,65^{\circ} \mathrm{C}\right)$ y lailuminanciasobrepasalarecomendablecon 306,99lx. La iluminancia en los corredores presenta diferencias marcadas, sin embargo, todos sobrepasan en promedio el índice ideal, por lo que todas las obras están propensas a deteriorarse a causa de sus efectos. 


\section{Deterioros encontrados}

La ubicación en los corredores no tiene una relación directa con el grado de deterioro que presentan ya que las condiciones son homogéneas en todo el patio. Las obras que presentan deterioros catalogados como nivel $2 \mathrm{y} 3$, requieren de un tratamiento pronto y especializado para evitar que estos empeoren y se ponga en riesgo la conservación de las mismas.

\section{Agente - indicador}

La humedad relativa es el factor de mayor incidencia, seguido porlatemperatura, y porúltimolailuminancia. Las variaciones dehumedad relativa producen cambios dimensionales del soporte, que lo inducen a pasarala fase plástica, en la cual se torna rígido y quebradizo, efecto evidenciado en deterioros presentes como ondulaciones, grietas, roturas y faltantes. Las bajas temperaturas han provocado deterioros como craquelados y pulverulencia. El desvanecimiento de color en las obras tiene relación con la intensidad de luz a la que están expuestas en el espacio estudiado.

\section{REFERENCIAS BIBLIOGRÁFICAS}

Czichos, H.; Saito, T. y Smith, L. (Editores) (2006). Handbook of metrology and testing. Springer

Herráez J., y Rodríguez M. (1989). Manual para el uso de aparatos y toma de datos de las condiciones ambientales en museos. Madrid: Dirección de Bellas Artes y Archivos

Instituto Nacional de Meteorología e Hidrología (Ecuador) (2008). Anuario Meteorológico 2006. Quito: SIGIHM. Recuperado de: http://www. serviciometeorologico.gob.ec/wp-content/ uploads/anuarios/meteorologicos/Am\%202006.pdf

Instituto Nacional de Meteorología e Hidrología (Ecuador) (2010). Anuario Meteorológico 2007. Quito: SIGIHM. Recuperado de: http://www. serviciometeorologico.gob.ec/wp-content/ uploads/anuarios/meteorologicos/Am\%202007.pdf

Instituto Nacional de Meteorología e Hidrología (Ecuador) (2010). Anuario Meteorológico 2008. Quito: SIGIHM. Recuperado de: http://www. serviciometeorologico.gob.ec/wp-content/ uploads/anuarios/meteorologicos/Am\%202008.pdf Instituto Nacional de Meteorología e Hidrología
(Ecuador) (2012). Anuario Meteorológico 2009. Quito: SIGIHM. Recuperado de: http://www. serviciometeorologico.gob.ec/wp-content/ uploads/anuarios/meteorologicos/Am\%202009.pdf

Instituto Nacional de Meteorología e Hidrología (Ecuador) (2012). Anuario Meteorológico 2010. Quito: SIGIHM. Recuperado de: http://www. serviciometeorologico.gob.ec/wp-content/ uploads/anuarios/meteorologicos/Am\%202010.pdf

Instituto Nacional de Meteorología e Hidrología (Ecuador) (2014). Anuario Meteorológico 2011. Quito: SIGIHM. Recuperado de: http://www. serviciometeorologico.gob.ec/wp-content/ uploads/anuarios/meteorologicos/Am\%202011.pdf

Instituto Nacional de Meteorología e Hidrología (Ecuador) (2015). Anuario Meteorológico 2012. Quito: SIGIHM. Recuperado de: http://www. serviciometeorologico.gob.ec/wp-content/ uploads/anuarios/meteorologicos/Am\%202012.pdf

Instituto Nacional de Patrimonio Cultural (Ecuador). ABACO: Sistema de información para la gestión del patrimonio cultural. Recuperado de: http://abacoweb.inpc.gob. ec:10080/inpc/InmInventarioBienPublic.seam

Instituto Nacional de Patrimonio Cultural. (2011) San Francisco, una bistoria para el futuro, Quito: INPC

Keyser, BW. (1984). Restraint without stress, history and prospects: a literature review of paintings as structures. Journal of the American Institute for Conservation. JAIC 1984, Volume 24, Number 1, Article 1 (pp. 01 to 13). Recuperado de: http://cool. conservation-us.org/jaic/articles/jaic24-01-001.html

Kollman, F. y Côté, W. (1968) Wood Science and Technology: Solid wood. Springer

Martiarena, X. (1992) Conservación y Restauración. Donostia: Eusko Ikaskuntza

Mecklenburg, M. (2007). Micro climates and moisture induced damage to paintings. En Tim Padfield y Karen Borchersen (Eds) Museum Microclimates, Contributions to the Copenhagen conference 19 - 23 November 2007. Recuperado de: http://www.conservationphysics. org / m m/mus mic/mus mic 150 . p d f

Michalski S. (2009) Humedad Relativa Incorrecta, 
Canadá: ICCROM. Recuperado de: http://www. cncr.cl/611/articles-56474_recurso_10.pdf

Roche, A. (2005). Comportement mécanique des peintures sur toile: Evaluation delastabilité mécanique aux variations d'humidité et de température. En Seminario internacional de conservación de pinturta. El soporte textil: comportamiento, deterioro y criterios de intervención. Valencia: Editorial delaUPV.

Saltos, E. (2014) Incidencia de los factores ambientales en las obras atribuidas a Miguel de Santiago ubicadas en el claustro del convento San Agustín. Quito: Universidad Tecnológica Equinoccial. Disponibleen: https://app.ute.edu.ec/sict/Biblioteca/ visor/abrir_pedido digital.aspx?mat_bib dig $\operatorname{cod}=1 \overline{07} 1632181 \overline{2} 5254205216196054 \overline{11} 613^{-}$ $015 \overline{3} 0$

04044136173 \&lectura $=1071632181252542052161$

96054116130153004044136173 \&imprimir $=$
064210163005051031046239187039202011108100 $078006 \&$ copiar $=064210163005051031046239187$ $039202011108100078006 \&$ guardar $=$

064210163005051031046239187039202011108100 $078006 \&$ catalogo $=1701631300030790751001470$ $87065039090187051112234 \&$ tpoTit $=06421016300$ $5051031046239187039202011108100078006 \& \mathrm{cA}$ SO

$\mathrm{Vc}=10716321812525420521619605411613015$

Vega, H. (2004) (Coordinador) Estudios para la restauración y consolidación del artesonado, cubierta y cúpulas de la iglesia de San Francisco. FONSAL, Municipio del Distrito Metropolitano de Quito. Quito, Ecuador. reg = 219223000017161042 017090009058099046195152191159\&mat_bib_ dig cod $=1071632181252542052161960541161301$ 53 